\title{
EL ÁLGEBRA LINEAL Y EL PROBLEMA DE MÁXIMOS Y MÍNIMOS
}

Santiago Relos Paco

\section{RESUMEN}

En este artículo se presenta una aplicación del álgebra lineal al problema de máximos y mínimos de funciones a varias variables. Se considera una función $f: U \subset \mathfrak{R}^{n} \rightarrow \mathfrak{R}, U$ abierto y $f$ dos veces diferenciable, se toma un punto $a \in U$ tal que $f^{\prime}(a)=0$, se plantea el problema de determinar si en este punto existe un máximo, mínimo o ninguna de estas situaciones. Se calcula $f^{\prime \prime}(a)=0$, como se sabe esta segunda derivada es una matriz simétrica en $M_{n, n^{\prime}}$, dependiendo de la signatura de $f^{\prime \prime}(a)=0$ se dará una respuesta al problema planteado.

Palabras Clave: Algebra Lineal, Máximos y Mínimos, Autovalores y Autovectores. 